\title{
Cognitive estimation of speed, movement and time across the lifespan
}

\author{
Jose L. Tapia ${ }^{1, \dagger}$, Francisco Rocabado ${ }^{1, \dagger}$, Jon Andoni Duñabeitia ${ }^{1,2, *}$ \\ ${ }^{1}$ Centro de Investigación Nebrija en Cognición, Universidad Nebrija, 28015 Madrid, Spain \\ ${ }^{2}$ Department of Language and Culture, The Arctic University of Norway, N-9037 Tromsø, Norway \\ "Correspondence: jdunabeitia@nebrija.es (Jon Andoni Duñabeitia) \\ ${ }^{\dagger}$ These authors contributed equally.
}

\section{DOI:10.31083/j.jin2101010}

This is an open access article under the CC BY 4.0 license (https://creativecommons.org/licenses/by/4.0/). Submitted: 26 March 2021 Revised: 15 April 2021 Accepted: 31 May 2021 Published: 28 January 2022

We investigated the role of age and gender in cognitive estimation abilities. Participants completed two online tasks, where different categories of estimation were assessed (speed estimation by time and distance, and auditory estimation). Data from a total of 18886 participants (9911 females) with ages between 18 and 65 years old were gathered. Results showed variations in the estimation capacity as a function of age, with a slow but progressive decline. Estimates of duration and sound seemed to be more affected by age than estimates of speed and movement. Overall, male participants showed a better performance on both auditory and visual estimation tasks compared to females.

\section{Keywords}

Cognitive estimation; Estimation abilities; Executive functions; Cognitive stimulation

\section{Introduction}

Cognitive estimation is defined as the ability to provide reasonable answers to questions for which relevant facts are known, but the exact answers are not readily available [1]. Estimation is about thinking of a new way to use our knowledge and experiences to find a plausible solution to a problem. Since the implementation of routine operations is not sufficient to generate plausible responses to these situations, estimation abilities entail several executive processes, including attention, planning, working memory, abstract reasoning, and self-correction. Cognitive estimation, therefore, requires identifying and selecting relevant information for a question or problem posed, retrieving and manipulating particular details, planning alternative answers, comparing these alternatives according to their relative probability of success, detecting improbable answers and correcting them, and finally choosing the most plausible answer [2]. In this line, estimation is better understood as a multifaceted set of cognitive mechanisms. In the current study we focused on the development of estimation skills circumscribed to predictive capacities associated with sensory information processing as a key feature of human adaptive behavior [3]. Estimation tasks that have a sensory-perceptual component (i.e., seeing a moving car and predicting its future location) involve a series of mental activities from domains related to attention, memory, processing load, imagery, abstract reasoning, judgment, and decision-making.

As seen, estimation is an important cognitive function for human functioning. Many daily situations depend on our ability to estimate time, distance, or speed. Even though most estimation processes may not seem of great relevance (e.g., estimating the shopping bill, the amount of food to prepare for a meal, the size of a shirt you want to buy for a gift, or if one will get to the bus on time), poor performance is likely to have a significant impact on a person's everyday [4]. Furthermore, failure in estimation abilities can lead to fatal consequences. For instance, incorrectly estimating other vehicles or pedestrians' distance or speed while driving or crossing the street may lead to a fatal accident. Therefore, it is essential to know the development of these skills to prevent an estimation failure and the consequences that may arise. Given the breadth of the concept of estimation and the various cognitive functions that may be involved, we narrowed our study down to the sensory-perceptual components, in line with the concepts of time-to-contact and time-to-arrival. Research supporting the hypothesis of a tight link between the decline in sensory function (mainly hearing and vision [5-7]) and a slowdown in cognitive skills $[8,9]$ also suggests that estimation abilities could also be affected as a function of age.

Speed and time estimation are relevant mechanisms for most living beings as they are essential for the interaction with external environment. Research on time perception has a long tradition and a dense literature background. A common relationship has been established between the estimation of time and age: as one gets older physical time is perceived as passing faster, thus existing a mismatch between real time and the subjective or internal experience [10]. Interestingly, several studies have suggested that women underestimate time lags when they are asked to produce or reproduce a time interval (namely, the answer they give is shorter than the actual elapsed time). Also, women have been shown to overestimate time lags when the task requires providing a verbal estimation (namely, their answer is longer 
than the actual elapsed time) [11-13]. Thus, scientific evidence shows greater accuracy and less variability in time estimation for males than for females, and these results have been typically explained in terms of the attentional resources allocated by each gender $[14,15]$. Similarly, in time-to-arrival estimation tasks that require the cognitive calculation of when an object will reach certain point in space, males have been found to perform more accurately than females, also supporting a possible gender difference in spatial reasoning skills $[16,17]$. Over the years, different methodological approaches have been used to investigate the experience of time. Some studies have used questionnaires asking participants whether they have noticed changes in their subjective perception of time with respect to earlier ages [18], while others include explicit tasks where individuals must estimate or reproduce the presentation time of a stimulus [19]. Overall, age, together with gender, have been two critical factors investigated in the last decades using online and offline methods to ascertain whether and how they modulate time estimation. Despite being studied for more than a century [20], results are blurred, and the real impact of age and gender in time estimation is still to be clarified. Recently, it has been proposed that the apparent contradictions between the outcomes of preceding studies could be due to the paradigms employed [21]. In prospective paradigms, where participants know beforehand that they will be asked about time passing, no significant gender differences are observed. On the other hand, in retrospective paradigms where participants do not know they will be asked to estimate the duration of the presented stimuli, males give more accurate duration estimations than females, who show poorer estimates and greater variability.

As seen, the literature on the development of estimation ability has a long history. The specific literature in arrival-time judgments started with Piaget's seminal works [22], studying the development of the ability to estimate time, movement and speed. According to his findings, estimation abilities are expected to be fully developed by the age of 11 . This same claim has been corroborated more recently. Dorfman [23] examined the timing and consequent anticipation of a target stimulus that disappeared from view shortly after initial movement and found that older age groups performed better than younger ones in samples with ages ranging between 6 and 19 years. Results also showed that males outperformed females, regardless of age. In the same line, Benguigui, Broderick and Ripoll [24] suggested that children are less efficient in estimating arrival-time than adults for longer occlusion durations; the longer an object was off-screen, the poorer children's estimation ability were. Notwithstanding, these studies focused on estimation skills' development at an early age but did not consider its development throughout life. Hence, while the seminal observation by Piaget may be correct at suggesting that estimation abilities are fully developed already at the age of 11 years, a general picture from young adulthood onwards is still missing. In this same line, it is worth noting that Harel et al. [25] found that although es- timation abilities in children experience significant improvement between 5 to 10 years, they did not find any ceiling effect for cognitive estimation. Quite on the contrary, they found that this ability kept improving until the age of 16 at a lower rate. It remains to be seen what the developmental course of these abilities from this age range is, and the current study was designed to provide evidence on this issue.

Estimation skills gain special relevance when considering the potential effects of aging on them. Traditionally it has been suggested that estimation (at the temporal level) operated either as a linear function [26] or as a square root function [27] concerning the past and present age. However, both hypotheses have subsequently been refuted, suggesting that the perceived estimate is relative to a linear proportion of the present age [28]. This approach, therefore, suggests agesusceptible effects on estimation abilities in non-pathological population. In this sense, it has been shown that in estimation tasks of a specific time interval, healthy older people present longer estimates and greater variability than younger adults [29]. The neuronal decline of dopaminergic functions and cortico-striatal circuits produced by aging could explain the aforementioned age-related deficiencies in the estimation processes [30]. Research on time-based prospective memory tasks has shown that older adults are less accurate at producing time estimates than younger adults in laboratory settings $[31,32]$ and environmental condition scenarios [33]. By way of illustration, the study by Waldum and McDaniel [33] compared the estimate of time elapsed during a game of trivia and found that older adults gave longer estimates than younger adults. These results are consistent with the notion of a slowing internal clock associated with age [34].

Estimation abilities are closely linked to executive functions, and the neural underpinnings of these skills have been classically located in the frontal lobes. Stemming from the patient reported by Shallice and Evans [1] with a massive right frontal lobe injury who showed a significant inability to produce adequate cognitive estimates, neuropsychological evidence has repeatedly endorsed the claim that estimation impairments are due to frontal executive dysfunction [35]. In this line, recent clinical and neurology literature corroborates these statements, frequently reporting impairments in judgment skills, problem-solving, and bizarre estimation responses after frontal lobe damage [36]. Not surprisingly, estimation skills have been consequently studied in a wide range of neurological pathologies such as dementia, Huntington's disease, Korsakoff's syndrome, Parkinson's disease, depression, or schizophrenia [4].

In light of the importance of adequately measuring these skills, several attempts have been made to develop tools to evaluate estimation abilities. One of the most used assessment tools is The Cognitive Estimation Test (CET), which is also useful for detecting frontal lobe lesions and executive dysfunction [2]. The CET evaluates processes such as reasoning, strategy planning, response plausibility, and general knowledge. Similarly, The Biber Cognitive Estima- 
tion Test (TBCET) contemplates four estimation categories: time/duration, quantity, weight, and distance. The TBCET also contributes to clinical research on patients with frontal or executive impairments, such as patients with attention deficit hyperactivity disorder and traumatic brain injury [37]. Another test that includes estimation skills among the cognitive domains and processes it explores is the Online General Cognitive Assessment Battery (CAB; CogniFit Inc., San Francisco, USA), with two specific tasks for this domain. The $\mathrm{CAB}$ has been validated against several major gold-standard neuropsychological tests [38] such as the Cambridge Neuropsychological Test Automated Battery, the Raven's Standard Progressive Matrices, the Wisconsin Card Sorting Test, the Continuous Performance Test, or the Stroop test. Validation studies have shown high correlations, good internal consistency in cognitive estimation evaluation, and an excellent test-retest reliability [39]. The use of the CAB is exponentially growing given that it sets the basis for effective cognitive stimulation programs [40].

Nonetheless, and despite the importance to develop widely used tools to assess estimation abilities and the need for normative data that correctly account for the potential differences as a function of age and gender, to date and to the best of our knowledge, no such description has been provided in the literature. In the present study, we aimed at systematically examining the effects of age and gender in cognitive estimation abilities associated with sensory information processingoperationalized as estimation of speed, movement, and sound duration (time)-in a large sample of more than 18000 healthy individuals using the two estimation tasks included in the CAB.

\section{Materials and methods \\ 2.1 Participants}

A total of 18886 healthy individuals aged between 18 and 65 years $(M=26.393, \mathrm{SD}=9.96)$ from 110 countries were recruited voluntarily through an online platform from January 2018 to June 2020. Participants were recruited through various collaborating institutions, universities, and research centers in different countries. Voluntary participants also joined the platform after hearing about it through social networks or from their relatives, friends or acquaintances. All participants gave informed consent. The institutional review board of the Universidad Nebrija approved the study with the code UNNE-2020-008. Exclusionary criteria included: (a) having a significant medical or neurological disorder such a stroke, multiple sclerosis, or Parkinson's disease; (b) having a psychiatric disorder such as depression or hyperactivity disorder; (c) having visual or auditory impairment; (d) having drug or alcohol abuse or dependence; (e) taking medication that affects central nervous system functioning.

\subsection{Procedure}

Participants completed two estimation tasks: visual speed estimation, and auditory estimation. To this end, participants used the CogniFit ${ }^{\circledR}$ (CogniFit Inc., San Francisco, USA) on- line platform. Each participant performed the tasks on their best schedule in an ecological environment (e.g., their home). Task presentation remained constant across participants.

\subsubsection{Estimation of speed and movement upon visual presentation.}

Estimation was measured based on the ability to calculate the speed of moving objects, the distance covered and to be covered, and how the joint interaction of speed and distance affects the motion of a moving object. Stimuli were presented on the screen, together with the response options, and participants had to select the correct answer. The task consisted of four stages, with four trials each. In stage 1, two moving balls (red and blue) were presented, and the participant had to determine which one moved faster with no visible path for the balls. A response time limit of 15 seconds was set. In stage 2, another ball (green) was added, and participants had to select which ball moved faster in a maximum time of 17 seconds. In stage 3 , another ball (yellow) was added, and participants had to indicate which ball moved twice as fast as a designated ball (the red one). The time limit was set to 19 seconds. In stage 4, while watching the four balls moving in four separate itineraries with visible paths, participants had to determine as quickly as possible which ball would arrive at a given point first. No time limit was set for this stage. The scoring in the four stages corresponded to the percentage of correct answers.

\subsubsection{Estimation of the duration of a continuous auditory stimulus.}

Estimation was measured based on the ability to interrupt an ongoing auditory stimulus so as to reproduce the exact length of time of a previously presented continuous auditory stimulus. The average time of each trial was fixed with a variation window of 0.3 seconds. Stimuli were harplike, bubbles-like, and piano-like sounds. The task included a learning trial and two assessment stages, with four trials each. In order to start the assessment stages, participants had to complete the learning stage. In stage 1 , the auditory stimulus was accompanied by visual support. In stage 2 , the visual stimuli remain still so that duration estimation proceeded exclusively based on auditory information. The score corresponded to the percentage of similarity between stimuli's duration (in milliseconds), and the duration of the stimuli stopped by the participants.

\subsection{Analysis}

Using the Nlem package (3.1-152, https://cran.r-project .org/package $=$ nlme) in RStudio (4.0.3, RStudio, Inc., https: //www.rstudio.com/), Linear Mixed-Effects Models analysis was conducted. Before model construction, data was wrangled on the same coding environment, and all estimation scores were transformed to z-scores. The age predictor was centered and scaled and coded using the deviation method, and the gender predictor was relabeled ( $1=$ male, $2=$ female).

An automated model selection process was followed in order to uncover the best fitting model structure by using the dredge function from the MuMln package (1.43.6, https: //CRAN.R-project.org/package=MuMIn). From the most 


\section{Predicted values of z-scores}

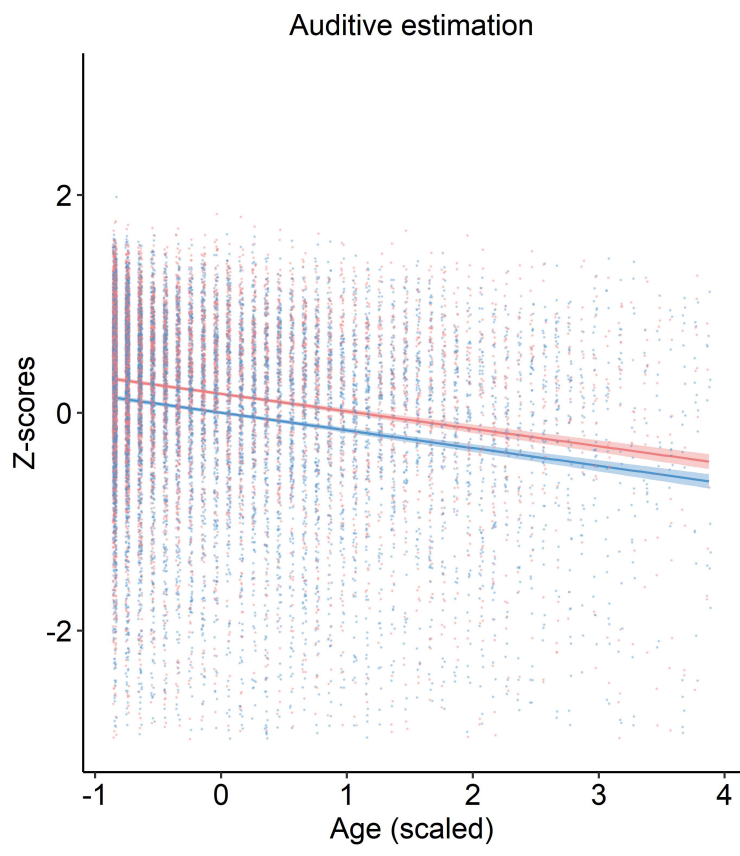

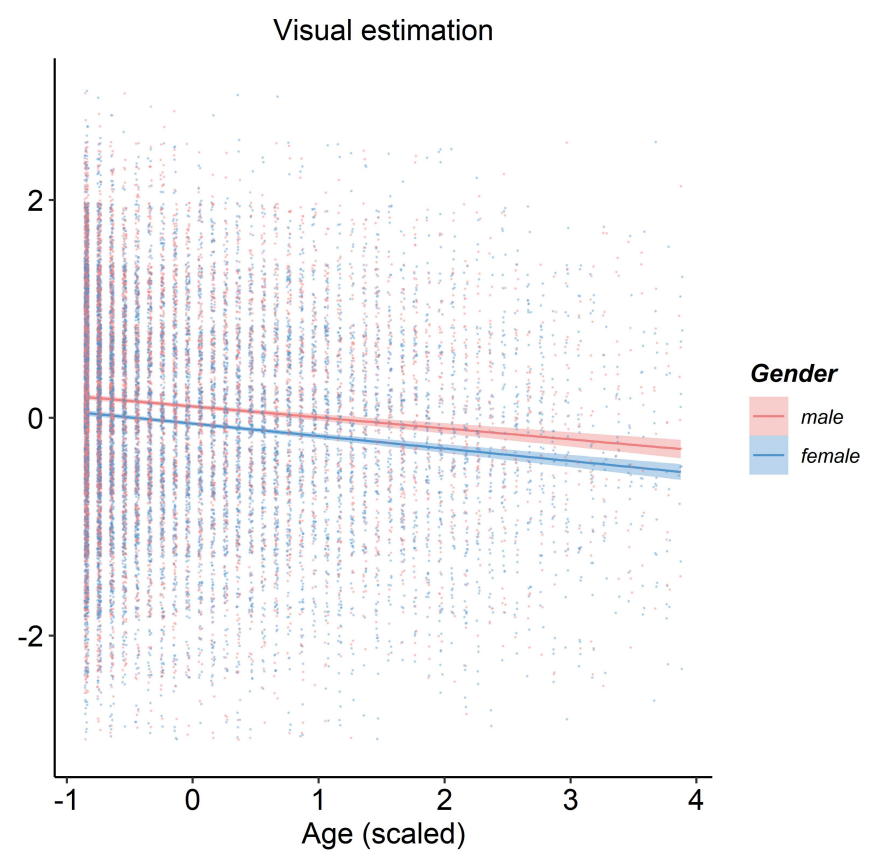

Fig. 1. Age and gender differences in estimation skills across modalities. The left panel corresponds to the estimation of the duration of continuous auditory stimuli presentation, and the right panel to the estimate of speed and movement upon visual stimuli presentation. The blue lines represent the predicted values for females, and the red lines represent the predicted values for males. Semi-transparent areas indicate the $95 \%$ confidence intervals of the fixed effect of estimation scores. The red dots are representative of the direct scores for males and the blue dots for females.

complex model structure and in an iterative process, the function compared all the possible model structure combinations of effects and interactions by their goodness-of-fit using the Akaike Information Criterion (AIC). From here, the simplest model capable of explaining participant estimation scores included Task (auditive | visual), Gender (1 | 2), Age and the interaction between Task and Gender as fixed effects, and Subject as random effect. The analysis of the best-fitting model was carried out in Jamovi (1.6.16, The jamovi Project, https://www.jamovi.org/).

\section{Results}

The sample was evenly split between males (48\%) and females (52\%), and it was demographically diverse, with 52\% from France, 10\% from the United States, 9\% from Russia, 5\% from Canada, and 24\% from over 100 other countries. Only 10 countries were represented by at least 250 participants.

All main effects were found significant. The effect of Task showed significant difference across modalities $(F(1,18884)$ $=3136.6, p<0.001$ ), with auditory estimation scores being higher than visual estimation scores ( $\mathrm{z}$-score auditory $=$ 58.8; $z$-score visual $=53.3$ ). The effect of Gender was also significant $(F(1,18883)=269.5, p<0.001)$. The scores of females were lower than those of males (z-score females = 55.1; $z$-score males $=57.2$ ). The main effect of Age was also significant $(F(1,18883)=845.1, p<0.001)$, showing that estimation scores declined as the age of the participants in- creased. As stated above, the interaction between Task and Age was significant $(\mathrm{F}(1,18884)=43.3, p<0.001)$, showing that while both visual and auditory estimation scores worsened with age, the effect was more pronounced for in the auditory modality. Follow-up comparisons confirmed this result (auditory modality: $\chi^{2}(1)=675, p<0.001$; visual modality: $\chi^{2}(1)=295, p<0.001$ ) (see Fig. 1).

\section{Discussion}

Estimation processes allow individuals to predict an object's future location based on its current speed, distance, and the corresponding time. These processes rely on a series of mechanisms tapping into complex cognitive functions that allow for a correct mapping between the actual contextual information and previous real-life situations. This way, estimation makes it possible for any person to carry out everyday activities avoiding obstacles and accidents without bumping into other people, as well as determining what will happen in the immediate future in a constantly active environment. Therefore, poor estimation abilities may impact everyday functioning. Multiple pathologies alter estimation, such as cranial trauma, brain tumor, Parkinson's disease, dementia, anxiety, depression, or schizophrenia. However, estimation ability can also be impaired in healthy populations because of aging and non-pathological cognitive decline. This being so, determining the course of cognitive estimation abilities could provide us with insights to understand the mech- 
anisms associated with them and to obtain possible markers of cognitive decline processes. The aim of the current study was to offer a general picture of the developmental course of estimation skills across gender and age ranges.

As we initially hypothesized, marked differences in the estimation skills were found as a function of age, with a slow but progressive decline that followed a linear function. Interestingly, estimation skills were shown to be differently affected by the age of the participants depending on the modality at test. Estimates of speed and movement based on visual information were less affected by age than estimates of duration and sound based on auditory information. Overall, and despite the modality differences shown, these findings demonstrate that estimation abilities begin to decline in the early adulthood. Significant differences were also observed as a function of gender, since male participants were consistently found to perform better in both tasks (auditory and visual) than female participants across ages. This gender effect was robust and stable, and did not vary across modalities, suggesting a generalized better estimation ability of males than females. While there is no unique explanation for the observed gender differences, we tentatively suggest that they could stem from the differences that have been previously reported between males and females in their perception of spatiotemporal information [17]. The evidence from preceding literature on gender differences in visuospatial perception and perceptual-motor skills supports this view and could potentially explain the observed effects $[18,41]$.

These results are consistent with the frontal aging hypothesis, which proposes that cognitive abilities mediated by the frontal lobe and its interconnecting neural circuitry are highly vulnerable to the effects of age [42]. As expected, the current study demonstrates that sensory-based estimation abilities change as a function of age, with a drop in performance from older as compared to younger samples. Nonetheless, it should be noted that the current study only considered age groups between 18 and 65 years, and future research should expand the age range to older participants to corroborate this linear trend. Hence, these data highlight the importance of implementing visual and auditory estimation assessment routines in cognitive evaluation batteries. Furthermore, these results point to the potential value of including tasks to train estimation skills in cognitive training programs, due to the importance that these cognitive abilities have in most daily activities. Future studies should be aimed at exploring the beneficial impact of early cognitive stimulation and remediation programs to prevent or compensate the impact of estimation dysfunction on the quality of life of the elderly.

\section{Abbreviations}

AIC, Akaike Information Criterion; CET, The Cognitive Estimation Test; TBCET, The Biber Cognitive Estimation Test.

\section{Author contributions}

JLT and JAD conceived and designed the research study; JLT performed the research; JLT, JAD and FR analyzed the data; JLT, JAD and FR wrote the manuscript. All authors contributed to editorial changes in the manuscript. All authors read and approved the final manuscript.

\section{Ethics approval and consent to participate}

The data analyzed in the currents study were obtained with the informed consent of all participants and fully anonymized in compliance with data protection laws. The institutional review board of the Universidad Nebrija approved the study with the code UNNE-2020-008.

\section{Acknowledgment}

The authors are very grateful to CogniFit Inc. for generously sharing their resources to make this study possible.

\section{Funding}

This study was supported by founding from the Government of Spain State Plan for Scientific and Technical Research and Innovation (FPU19/02239, PGC2018-097145-BI00 and RED2018-102615-T) and the Community of Madrid (H2019/HUM-5705).

\section{Conflict of interest}

The authors declare no conflict of interest. None of the authors are employees of CogniFit Inc.

\section{References}

[1] Shallice T, Evans ME. The involvement of the frontal lobes in cognitive estimation. Cortex. 1978; 14: 294-303.

[2] MacPherson SE, Wagner GP, Murphy P, Bozzali M, Cipolotti L, Shallice $\mathrm{T}$. Bringing the cognitive estimation task into the $21 \mathrm{st}$ century: Normative data on two new parallel forms. PLoS ONE. 2014; 9: e92554.

[3] Birman D, Gardner JL. A flexible readout mechanism of human sensory representations. Nature Communications. 2019; 10: 3500.

[4] Fortune DG, Richards HL. Assessing cognitive estimation and its effects on community integration in people with acquired brain injury undergoing rehabilitation. BioMed Research International. 2017; 2017: 2874819.

[5] Fozard JL. Vision and hearing in aging. Handbook of the Psychology of Aging. 1990; 70: 150-170.

[6] Allison T, Hume AL, Wood CC, Goff WR. Developmental and aging changes in somatosensory, auditory and visual evoked potentials. Electroencephalography and Clinical Neurophysiology. 1984; 58: 14-24.

[7] Price D, Tyler LK, Neto Henriques R, Campbell KL, Williams N, Treder MS, et al. Age-related delay in visual and auditory evoked responses is mediated by white- and grey-matter differences. Nature Communications. 2017; 8: 15671.

[8] Spear PD. Neural bases of visual deficits during aging. Vision Research. 1993; 33: 2589-2609.

[9] Bruffaerts R, Tyler LK, Shafto M, Tsvetanov KA, Clarke A. Perceptual and conceptual processing of visual objects across the adult lifespan. Scientific Reports. 2019; 9: 13771.

[10] Fraisse P. Perception and estimation of time. Annual Review of Psychology. 1984; 35: 1-36.

[11] Hancock PA, Arthur EJ, Chrysler ST, Lee J. The effects of sex, tar- 
get duration, and illumination on the production of time intervals. Acta Psychologica. 1994; 86: 57-67.

[12] Hancock PA, Rodenburg GJ, Mathews WD, Vercruyssen M. Estimation of duration and mental workload at differing times of day by males and females. Proceedings of the Human Factors Society Annual Meeting. 1988; 32: 857-861.

[13] Kirkcaldy BD. Individual differences in time estimation. International Journal of Sport Psychology. 1984; 15: 11-24.

[14] Espinosa-Fernández L, Miró E, Cano M, Buela-Casal G. Agerelated changes and gender differences in time estimation. Acta Psychologica. 2003; 112: 221-232.

[15] Brown SW. Time perception and attention: the effects of prospective versus retrospective paradigms and task demands on perceived duration. Perception \& Psychophysics. 1985; 38: 115-124.

[16] Rusch ML, Schall MC, Lee JD, Dawson JD, Edwards SV, Rizzo M. Time-to-contact estimation errors among older drivers with useful field of view impairments. Accident Analysis \& Prevention. 2016; 95: 284-291.

[17] Schiff W, Oldak R. Accuracy of judging time to arrival: effects of modality, trajectory, and gender. Journal of Experimental Psychology: Human Perception and Performance. 1990; 16: 303-316.

[18] Joubert CE. Structured time and subjective acceleration of time. Perceptual and Motor Skills. 1984; 59: 335-336.

[19] Craik FIM, Hay JF. Aging and judgments of duration: Effects of task complexity and method of estimation. Perception \& Psychophysics. 1999; 61: 549-560.

[20] Seashore CE. Some psychological statistics II. The material weight illusion (pp. 36-46). University of Iowa Studies in Psychology: United States of America. 1899.

[21] Block RA, Hancock PA, Zakay D. Sex differences in duration judgments: a meta-analytic review. Memory \& Cognition. 2000; 28: 1333-1346.

[22] Piaget J. The child's conception of movement and speed. GET Holloway and MJ Mackenzie, (Trans.) Rout ledge and Kegan Paul: London. 1946.

[23] Dorfman PW. Timing and anticipation: a developmental perspective. Journal of Motor Behavior. 1977; 9: 67-79.

[24] Benguigui N, Broderick M, Ripoll H. Age differences in estimating arrival-time. Neuroscience Letters. 2004; 369: 197-202.

[25] Harel BT, Cillessen AHN, Fein DA, Bullard SE, Aviv A. It takes nine days to iron a shirt: the development of cognitive estimation skills in school age children. Child Neuropsychology. 2007; 13: $309-318$.

[26] Janet P. Une illusion d'optique interne. Revue Philosophique de la France et de l'Étranger (pp. 497-502). Presses Universitaires de France: France. 1877. (In French)

[27] Lemlich R. Subjective acceleration of time with aging. Perceptual and Motor Skills. 1975; 41: 235-238.
[28] Gallant R, Fidler T, Dawson KA. Subjective time estimation and age. Perceptual and Motor Skills. 1991; 72: 1275-1280.

[29] Turgeon M, Wing AM. Late onset of age-related difference in unpaced tapping with no age-related difference in phase-shift error detection and correction. Psychology and Aging. 2012; 27: 11521163.

[30] Turgeon M, Lustig C, Meck WH. Cognitive aging and time perception: roles of Bayesian optimization and degeneracy. Frontiers in Aging Neuroscience. 2016; 8: 102.

[31] Einstein GO, McDaniel MA, Richardson SL, Guynn MJ, Cunfer AR. Aging and prospective memory: examining the influences of self-initiated retrieval processes. Journal of Experimental Psychology: Learning, Memory, and Cognition. 1995; 21: 996-1007.

[32] Park DC, Hertzog C, Kidder DP, Morrell RW, Mayhorn CB. Effect of age on event-based and time-based prospective memory. Psychology and Aging. 1997; 12: 314-327.

[33] Waldum ER, McDaniel MA. Why are you late? Investigating the role of time management in time-based prospective memory. Journal of Experimental Psychology: General. 2016; 145: 10491061.

[34] Vanneste S, Pouthas V, Wearden JH. Temporal Control of Rhythmic Performance: a Comparison between Young and Old Adults. Experimental Aging Research. 2011; 27: 83-102.

[35] Harrington DL, Castillo GN, Greenberg PA, Song DD, Lessig S, Lee RR, et al. Neurobehavioral mechanisms of temporal processing deficits in Parkinson's disease. PLoS ONE. 2011; 6: e17461.

[36] Cipolotti L, MacPherson SE, Gharooni S, van-Harskamp N, Shallice T, Chan E, et al. Cognitive estimation: performance of patients with focal frontal and posterior lesions. Neuropsychologia. 2018; 115: 70-77.

[37] Bullard SE, Fein D, Gleeson MK, Tischer N, Mapou RL, Kaplan E. The Biber cognitive estimation test. Archives of Clinical Neuropsychology. 2004; 19: 835-846.

[38] Haimov I, Hanuka E, Horowitz Y. Chronic insomnia and cognitive functioning among older adults. Behavioral Sleep Medicine. 2008; 6: 32-54.

[39] CogniFit. Researchers Assessment Battery: Validity description of CogniFit assessments. Cognifit. 2016.

[40] Tapia JL, Duñabeitia JA. Improving language acquisition and processing with cognitive stimulation. Frontiers in Psychology. 2021; 12: 663773 .

[41] Rusch ML, Schall MC, Lee JD, Dawson JD, Rizzo M. Augmented reality cues to assist older drivers with gap estimation for leftturns. Accident Analysis \& Prevention. 2014; 71: 210-221.

[42] Greenwood PM. The frontal aging hypothesis evaluated. Journal of the International Neuropsychological Society. 2000; 6: 705726. 